Document downloaded from:

http://hdl.handle.net/10251/84407

This paper must be cited as:

García Miquel, AH.; Barrera Vilar, D.; Amat, R.; Kurlyandskaya, GV.; Sales Maicas, S. (2016). Magnetic actuator based on giant magnetostrictive material Terfenol-D with strain and temperature monitoring using FBG optical sensor. Measurement. 80(2):201-206. doi:10.1016/j.measurement.2015.11.035.

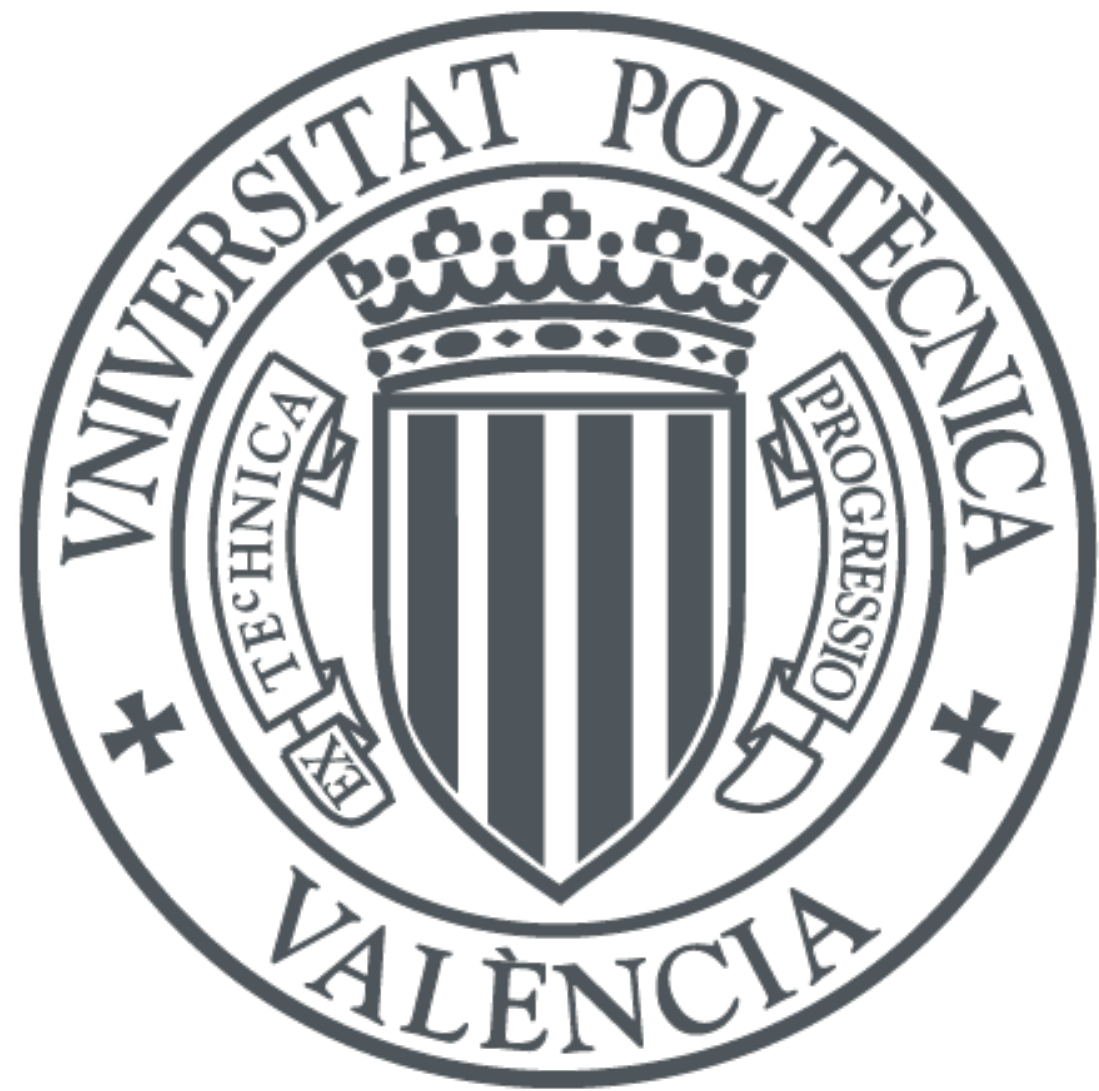

The final publication is available at

http://dx.doi.org/10.1016/j.measurement.2015.11.035

Copyright Elsevier

Additional Information 


\title{
Magnetic actuator based on giant magnetostrictive material Terfenol-D with strain and temperature monitoring using FBG optical sensor
}

\author{
H. García-Miquel ${ }^{1,2}$, D. Barrera ${ }^{1}$, R. Amat ${ }^{1}$, G.V. Kurlyandskaya ${ }^{3,4}$, S. Sales ${ }^{1}$ \\ ${ }^{1}$ ITEAM Research Institute, Universitat Politécnica de Valencia, C/Camino de Vera s/n, E-46022 Valencia, Spain. \\ ${ }^{2}$ Department of Electronic Engineering, Universitat Politécnica de Valencia, C/Camino de Vera s/n, E-46022 \\ Valencia, Spain. \\ ${ }^{3}$ Department of Electricity and Electronics, University of Basque Country UPV-EHU, 48080 Bilbao, Spain. \\ ${ }^{4}$ Ural Federal University, 620000 Ekaterinburg, Russia
}

\begin{abstract}
We have designed a temperature and strain monitoring system for a magnetic actuator based on the giant magnetostrictive material Terfenol-D ( $\mathrm{Tb}_{0.3} \mathrm{Dy}_{0.7} \mathrm{Fe}_{1.92}$ ) with Fiber Bragg grating (FBG) sensors. Magneto-elastic properties of Terfenol-D depend on magnetization, stress pre-history, and temperature. In order to simultaneously monitor these effects, we have implemented a system based on a cylindrical Terfenol-D rod monitored with four FBGs that allows making the appropriate compensations on the strain measurement due to temperature drift. We have measured the magnetostriction in the axial and the transverse directions for the Terfenol-D rod with two perpendicular FBGs, and calculated the Poisson ratio. An additional mechanical system for strain amplification has been designed in order to increase sensitivity, by a factor of 4, in the strain measurement with the FBG sensor.
\end{abstract}

Keywords: Magneto Optic Sensors, Magnetic Optic Actuator, Magnetostriction, Fiber Bragg Grating, Terfenol-D, Fiber Optic.

\section{Introduction}

Magnestrostictive materials have been widely used for the construction of magnetic sensors and actuators [1,2] Among other materials, Terfenol-D (Tb0.3 Dy0.7 Fe1.92) can be considered as the most studied one due to the possibility to achieve high magnetostriction strains [3,4,]. It is widely known that the magneto-elastic properties of this material can be modified as a function of the combination of both magnetization and stress pre-history. However, other parameters such as temperature may affect the optimum value of the operation point of Terfenol-D based sensors and actuators. In order to study the role of all these factors simultaneously one can use optical fiber sensors. They are a good option due to the small size, their intrinsically safe operation based on the use of light, the resistance to hard environmental conditions or their insensitivity to any external electromagnetic interference.

Fiber Bragg gratings (FBGs) is one of the most important optical fiber sensor type. These devices offer the possibility to implement several sensors in the same optical

Corresponding author: H. García-Miquel (hgmiquel@eln.upves). fiber and they are characterized by a relatively simple interrogation method [6]. For the above mentioned reasons FBGs have been used in combination with Terfenol-D to implement magnetic field sensors and actuators with very different operation designs [7-12].

FBGs are periodic or quasi-periodic modulations of the refractive index of the core of the optical fiber. This modulation of the refractive index is commonly produced by exposing the optical fiber to a high intensity ultra violet light pattern [13]. The periodic modulation of the refractive index causes the light to be reflected at certain wavelengths while it remains near transparent for the rest of the optical spectrum. The wavelength at which the maximum reflectivity is produced is known as the Bragg wavelength. It is given by $\lambda_{B}=2 n_{\text {eff }} \Lambda$, where $n_{\text {eff }}$ is the effective refractive index of the fiber core and $\Lambda$ is the physical period of the induced refractive index modulation. Under strain or temperature changes the Bragg wavelength suffers a shift that can be expressed as the sum of the effects of these two contributions:

$$
\begin{gathered}
\Delta \lambda_{B}=\frac{\partial \lambda_{B}}{\partial \varepsilon} \varepsilon+\frac{\partial \lambda_{B}}{\partial T} \Delta T \\
\frac{\partial \lambda_{B}}{\partial \varepsilon}=\lambda_{B}\left[1-\frac{n_{e f f}^{2}}{2}\left[p_{12}-v\left(p_{11}+p_{12}\right)\right]\right]
\end{gathered}
$$




$$
\frac{\partial \lambda_{B}}{\partial T}=\lambda_{B}\left[\alpha_{\Lambda}+\frac{1}{n_{\text {eff }}} \frac{\partial n_{\text {eff }}}{\partial T}\right]
$$

The first term represents the strain dependence, where $p_{11}$ and $p_{12}$ are the coefficients of the strain-optic tensor, $v$ is the Poisson ratio and $\varepsilon$ is the strain. The second term shows the temperature dependence, where $\alpha_{\Lambda}$ represents the thermal expansion coefficient of the optical fiber and $\partial n_{\text {eff }} / \partial T$ represents the change in the effective refractive index with temperature.

In this work we have designed, fabricated and studied a magnetic actuator monitored with fiber Bragg gratings under different conditions: temperature, stress, and external magnetic field.

\section{Experimental}

A cylindrical Terfenol-D rod of $5 \mathrm{~cm}$ long and $13 \mathrm{~mm}$ in diameter has been used as the magnetic actuator core. Four FBGs of $2 \mathrm{~mm}$ length were fabricated using a phase mask method and a doubled Argon-ion laser with a wavelength of $244 \mathrm{~nm}$. The full width at half maximum of the FBGs is approximately $0.35 \mathrm{~nm}$ for all the FBGs and they have central wavelength values of $1529.3 \mathrm{~nm}, 1545.3 \mathrm{~nm}, 1551.2 \mathrm{~nm}$ and $1566.9 \mathrm{~nm}$. Figure 1a shows the spectrum of one of the FBGS used during the experimental tests. The first FBG was axially glued (with ethyl cyanoacrylate glue) to the surface of the Terfenol-D rod while the second one was glued in the perpendicular direction. A third FBG was fixed to a strain amplification structure in order to obtain higher sensitivity in the strain measurement. Finally, the fourth FBG was attached with a silica thermal paste in contact with the surface with the aim of measuring and compensating the thermal effects (offset and sensitivity drift). Fig. 2 shows the general scheme of the magnetic actuator with the FBG monitoring system and the strain amplification structure.

The strain amplification structure consists of the Terfenol-D rod with two structural units made of brass at both ends of the magnetrostictive material. A FBG is placed into the gap between the two brass pieces allowing the magnification of the strain measurement with respect to the strain in the Terfenol-D rod.

The sample was placed inside a water cooled solenoid connected to a programmable current supply which creates the axial magnetic field (see Fig.1b). The solenoid (creating a constant magnetic field) with $19.25(\mathrm{kA} / \mathrm{m}) / \mathrm{A}$ constant was fed by a bipolar current supply KEPCO 72-14. The generated magnetic field was measured by the SYPRIS 7010 Gaussmeter. The optical sensors were monitored using a Micron Optics SM125 FBG interrogator. The behavior of the magnetic actuator was studied under different conditions of stress, temperature and magnetic field.
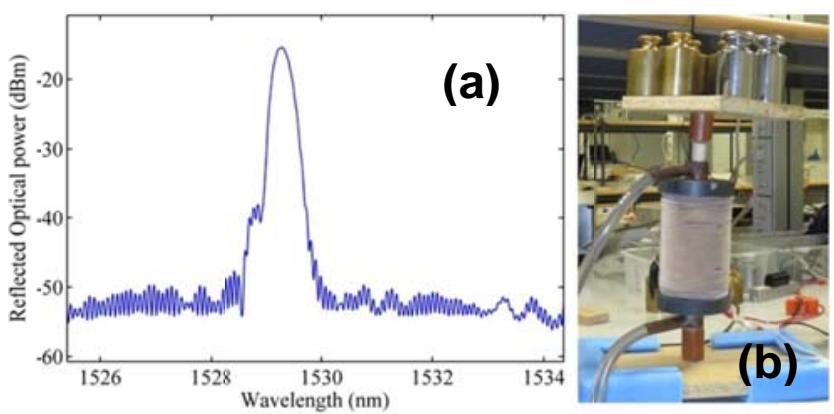

Fig. 1. (a) FBG spectrum, (b) water cooled solenoid for a magnetic field generation with compression load.

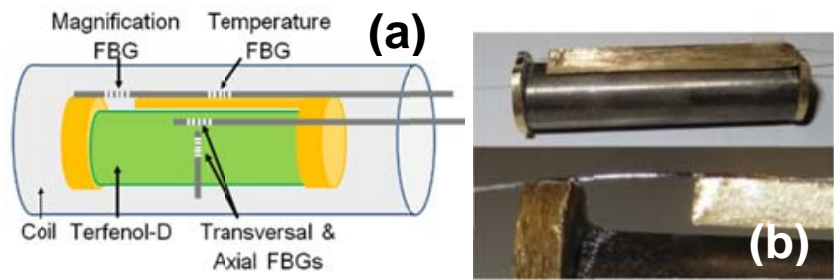

Fig. 2. (a) Schematic of the magnetostrictive actuator with FBG optical sensors, (b) image of the Terfenol D with the FBG and the strain amplification structure.

\section{Results and discussion}

In order to design a magnetostrictive actuator with Terfenol-D, we have investigated the effect of a compression stress on the Terfenol-D, the response of strain against magnetic field, and the temperature effect on strain and sensitivity. Finally, we have designed a strain amplification structure to obtain a better sensitivity and accuracy in the strain measurement.

The giant magnetostrictive material Terfenol-D modifies its magneto-elastic properties as a function of the combination of both magnetization and stress pre-history. We have studied the effects of the stress under static magnetic fields. Compression stresses were applied to the Terfenol-D rod actuator by means of $1 \mathrm{~kg}(73.8 \mathrm{kPa} / \mathrm{kg})$ weight loading progressively increased up to $9 \mathrm{~kg}$ and then reloaded down to the initial condition. Fig. 3a shows the strain measured by the axial FBGs under no applied magnetic field. In order to recreate the same initial conditions of measurement, we have applied a $96 \mathrm{kA} / \mathrm{m}$ pulse in the axial direction of the Terfenol$\mathrm{D}$ rod (see the inset of Fig.3a). Fig. 3b shows the results for a static magnetic field of $48 \mathrm{kA} / \mathrm{m}$. It can be seen that in the first case there is a clear hysteretic behavior while in the presence of a magnetic field this hysteresis is almost negligible. The application of a static magnetic field also 
reduces the stress sensitivity almost by a factor of 2 . The observed behavior is a consequence of the contribution of two effects: one contribution is due to the compression stress that leads to an alignment of the magnetization in the transverse direction (because of the magnetoelasticity) and the second one is consequence of the axial magnetic field aligning the magnetization in the axial direction of the rod.
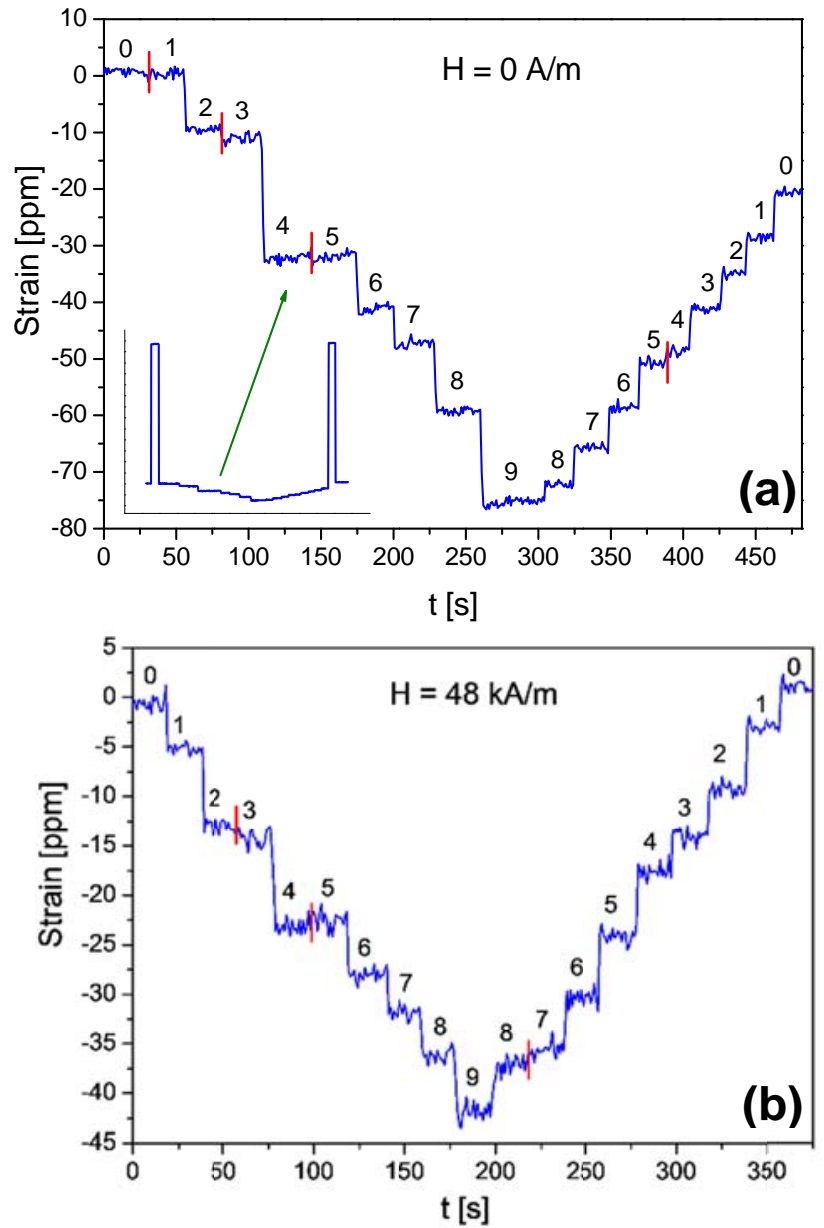

Fig. 3. Strain measured on Terfenol-D for and increasing/decreasing compression stress. The numbers represent the number of $\mathrm{kg}$ placed over Terfenol-D road (73.8 $\mathrm{kPa} / \mathrm{kg}$ ): (a) without applied magnetic field, (b) with an applied magnetic field of $48 \mathrm{kA} / \mathrm{m}$.

Next step in this work was to obtain the actuator strain response versus the applied magnetic field. For that matter we applied a quasi-static triangular magnetic field of $0.01 \mathrm{~Hz}$ to the Terfenol-D rod and measured the longitudinal and transverse strain related to a magnetostriction phenomenon. Fig. 4 shows the longitudinal and transverse magnetostriction produced by Terfenol-D when the amplitude of the magnetic field is increased. Fig. 5 shows a detailed view of the magnetostriction measured for a triangular magnetic field of $125 \mathrm{kA} / \mathrm{m}$ amplitude. From the longitudinal strain $\varepsilon_{\text {long }}$ and the transverse strain $\varepsilon_{\text {trans }}$, of magnetostriction origin, we have obtained the Poisson ratio:

$$
v=-\frac{\varepsilon_{\text {trans }}}{\varepsilon_{\text {long }}}=0.29
$$

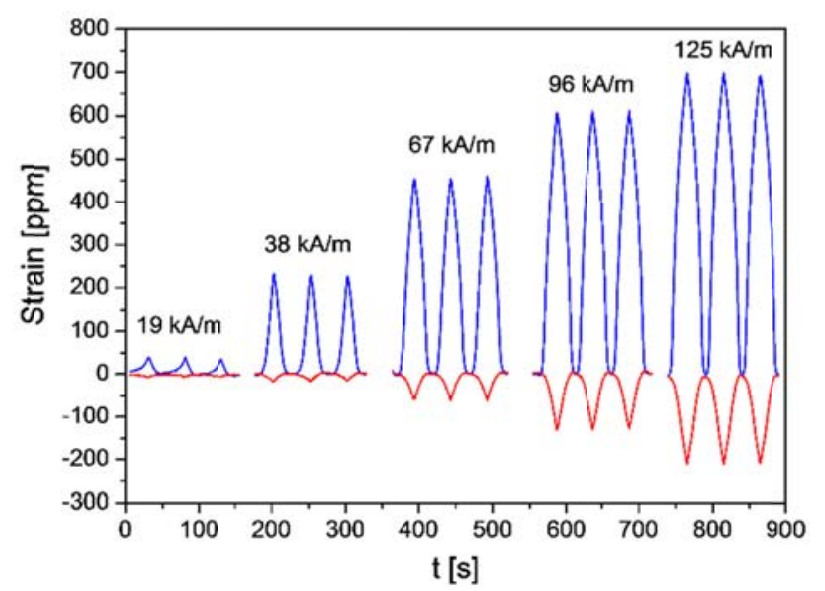

Fig. 4. Longitudinal (blue) and transverse (red) magnetostriction of Terfenol-D for a triangular magnetic field of $0.01 \mathrm{~Hz}$ frequency and different amplitude values.

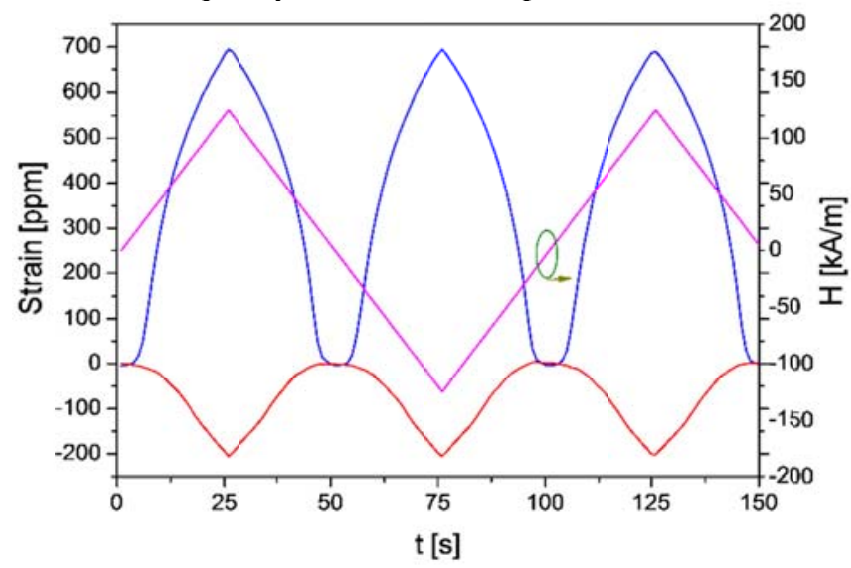

Fig. 5. Longitudinal (blue) and transverse (red) magnetostriction of Terfenol-D for a triangular magnetic field (magenta) of $0.01 \mathrm{~Hz}$ frequency and $125 \mathrm{kA} / \mathrm{m}$ amplitude.

Fig.6 shows the magnetostriction versus magnetic field for different values of compression stress. It can be seen that the magnetostriction increases with a compression stress.

The magnetostriction was measured for an increasing and decreasing magnetic field in order to evaluate the magnetostriction hysteresis (Fig.7) for magnetic field amplitude of $125 \mathrm{kA} / \mathrm{m}$. The strain in Terfenol has not a linear behavior with magnetic field, but it can be fit with a 5 grade polynomial fit (see Fig.8). Fig.9 shows the derivative of the strain with respect to a magnetic field (se also Fig.8), that is the sensitivity of strain versus magnetic field. According to these data, the magnetic field to apply to the actuator makes sense in the interval of $12 \mathrm{kA} / \mathrm{m}$ to $125 \mathrm{kA} / \mathrm{m}$. The sensitivity reaches the maximum for $40 \mathrm{kA} / \mathrm{m}$ with sensitivity of 13,3 
$\mathrm{ppm} /(\mathrm{kA} / \mathrm{m})$.

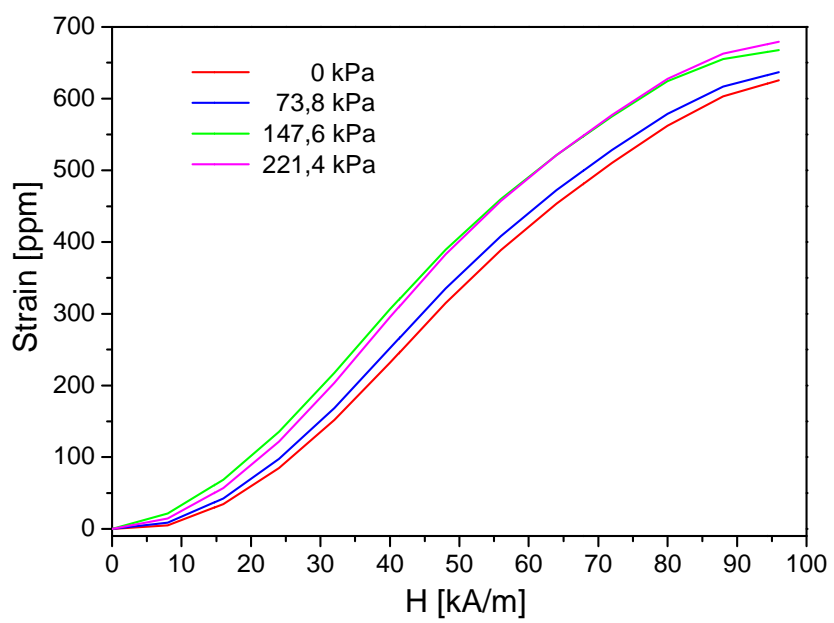

Fig.6: Strain versus magnetic field for different compression stress.

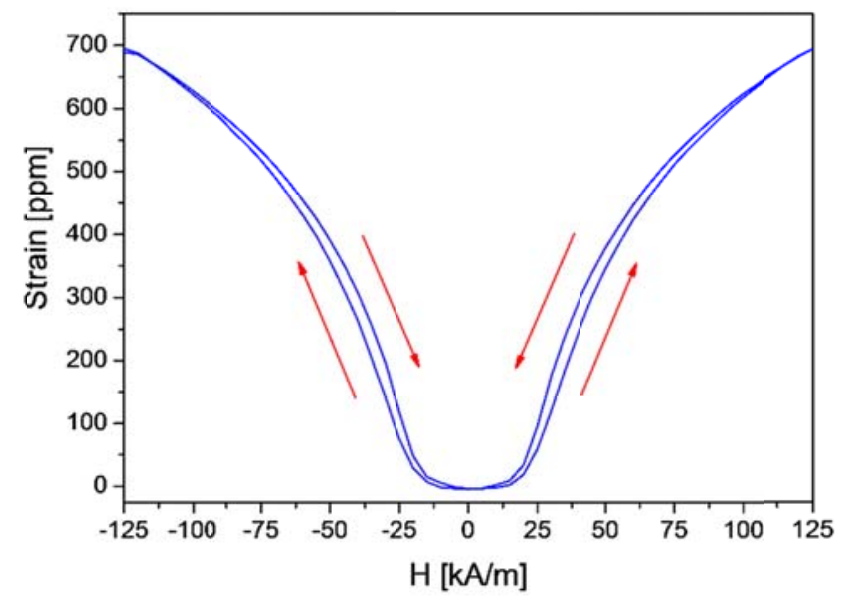

Fig. 7. Longitudinal magnetostriction of Terfenol-D rod as a function of applied magnetic field for up and down sweep, showing the hysteresis of magnetostriction.

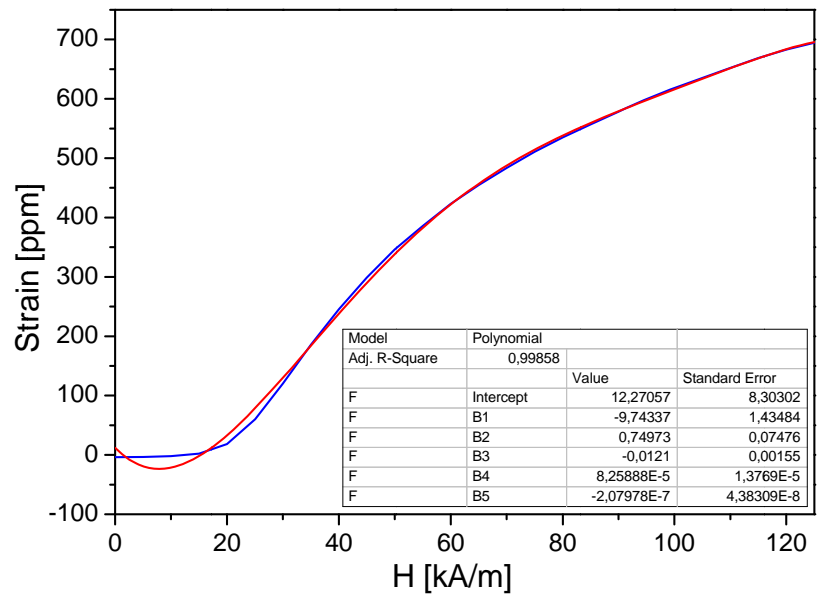

Fig. 8. Polynomial fit of longitudinal magnetostriction of Terfenol-D rod as a function of applied magnetic field.

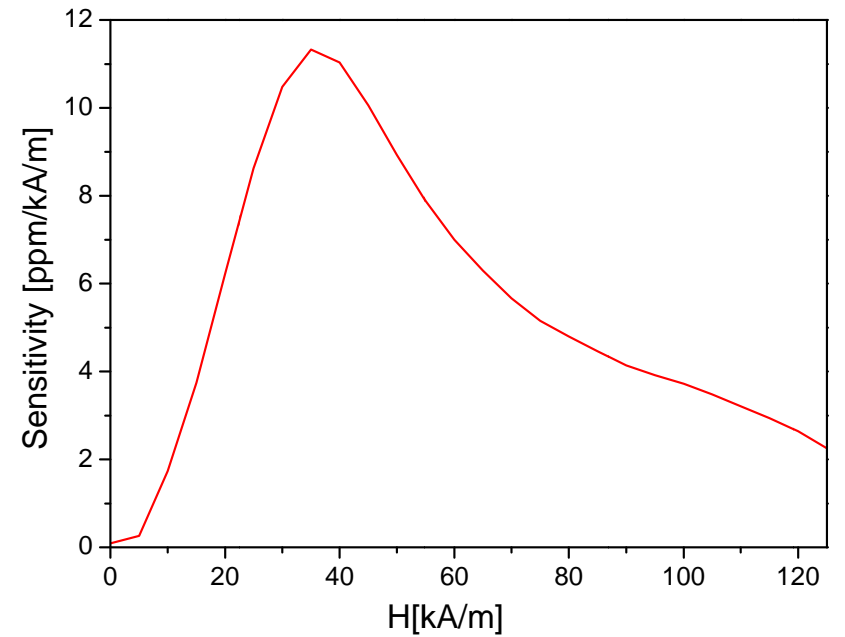

Fig. 9. Longitudinal mangetostriction sensitivity of Terfenol$\mathrm{D}$ rod as a function of applied magnetic field (for down field sweep).

For the development of a precise actuator based on Terfenol-D it is very important to take into account the thermal effects to distinguish it from magnetostriction. Terfenol-D rod suffers a strain response with a temperature change, and also a change in the magnetostriction sensitivity. Measurements at constant temperature have been made with water cooled solenoid. We used the FBG attached to the Terfenol-D rod with silica thermal paste in order to measure the temperature drift (with $9.1 \mathrm{pm} /{ }^{\circ} \mathrm{C}$ sensitivity). Fig. 10 shows the thermal effects produced when a triangular magnetic field of $96 \mathrm{kA} / \mathrm{m}$ amplitude is applied and the cooling pump for the solenoid water refrigeration is turned off, producing a temperature increase in the Terfenol-D rod.

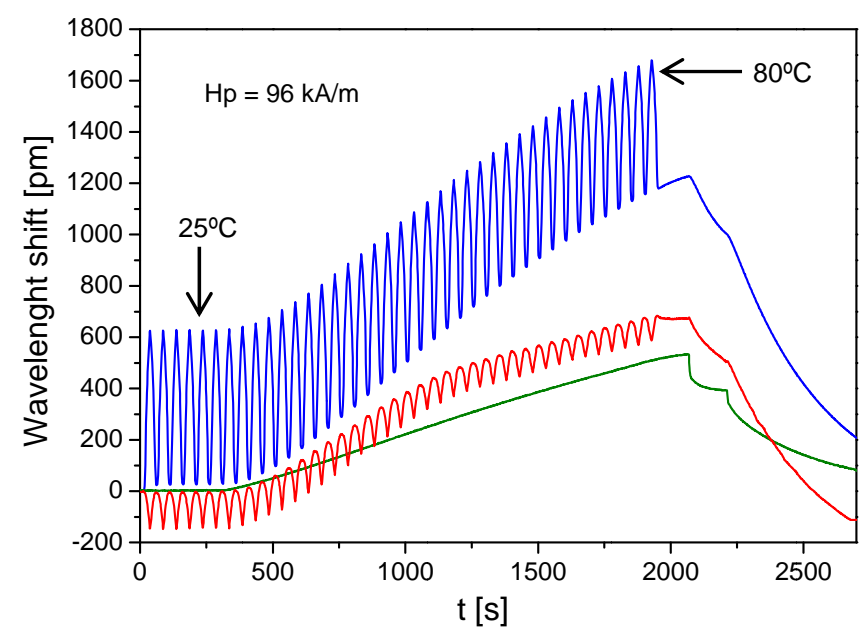

Fig. 10. Wavelength shift due to longitudinal (blue) and transverse (red) magnetostricction and thermal drift of the Terfenol-D rod in a triangular magnetic field of $\mathrm{Hp}=96 \mathrm{kA} / \mathrm{m}$ amplitude and $0.01 \mathrm{~Hz}$. Wavelength shift of the FBG (green) that is used for temperature measurement $\left(9.1 \mathrm{pm} /{ }^{\circ} \mathrm{C}\right)$. 
The measured magnetostriction value shifts as a consequence of the thermal expansion of the involved materials. It can be observed that the wavelength shift produced by the temperature drift due to the thermal expansion coefficient of Terfenol-D is higher than the magnetostriction drift, justifying the necessity of temperature measurement for the right compensation of the thermal effects. The initial temperature was $25^{\circ} \mathrm{C}$ and the maximum reached temperature was $80^{\circ} \mathrm{C}$. As temperature increased there was a reduction of the magnetostriction sensitivity, that can be observed as a reduction in the excursion amplitude of the measured strain; $600 \mathrm{pm}$ at $25^{\circ} \mathrm{C}$ to $500 \mathrm{pm}$ at $80^{\circ} \mathrm{C}$ (see fig.10).

The wavelength shift measured by the FBG due to the thermal drift of Terfenol-D was also measured applying a constant current of 14 A through the Terfenol-D rod that produced an increase of the temperature. Fig.11 shows the thermal drift of Terfenol-D and the temperature measurement with the FGB (sensitivity of $9.1 \mathrm{pm} /{ }^{\circ} \mathrm{C}$ ). The temperature varies from $25^{\circ} \mathrm{C}$ to $80^{\circ} \mathrm{C}$, and then decreases again down to $25^{\circ} \mathrm{C}$ once the applied current is cut off. While the FBG attached to the actuator by the thermal paste has a sensitivity of $9.1 \mathrm{pm} /{ }^{\circ} \mathrm{C}$, the FBG glued onto the Terfenol-D rod has a sensitivity of $22.7 \mathrm{pm} /{ }^{\circ} \mathrm{C}$, that is 2.5 times higher than the sensitivity of FBG which is used as a temperature sensor. There is a delay between the two FBGs signals (see Fig. 11) caused by the thermal conductivity of the glue used for the connection of the FBG and the Terfenol-D rod.

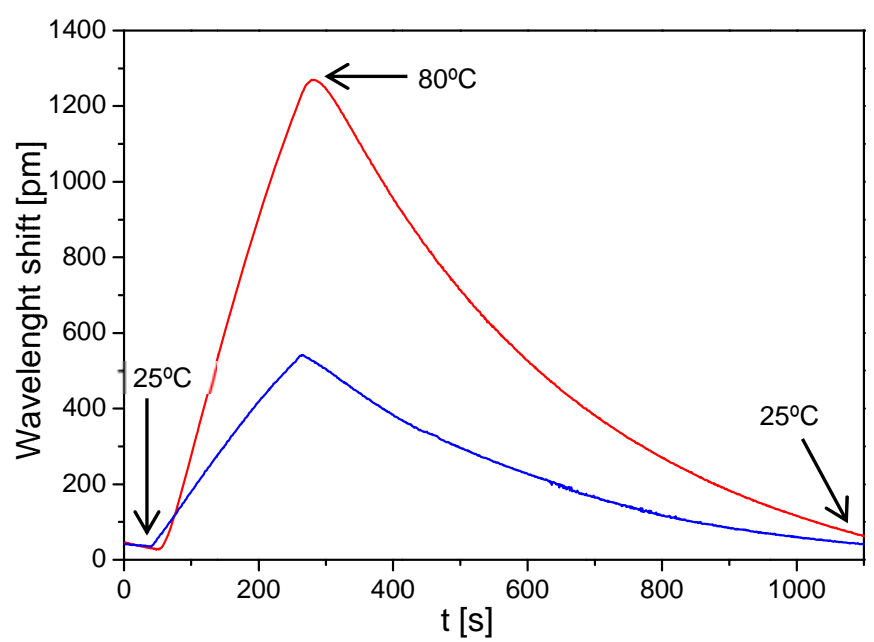

Fig. 11. Thermal drift of longitudinal magnetostriction (red) when the Terfenol is heated by a current. Wavelength shift of an FBG due to temperature drift (blue) used for temperature measurement $\left(9.1 \mathrm{pm} /{ }^{\circ} \mathrm{C}\right)$.

In order to measure the strain with higher sensitivity we have designed a strain amplifier (see Fig.1). With the use of a simple amplification construction it was possible to amplify the signal by a factor of 4 . Fig. 12 shows the longitudinal strain measured with FBG glued to the surface of the Terfenol-D rod (blue) and the strain measured with the FBG connected to the amplifying construction (red). A triangular magnetic field of $0.01 \mathrm{~Hz}$ and amplitudes of $38.5 \mathrm{kA} / \mathrm{m}$ to 125 $\mathrm{kA} / \mathrm{m}$ were applied for the measurements.

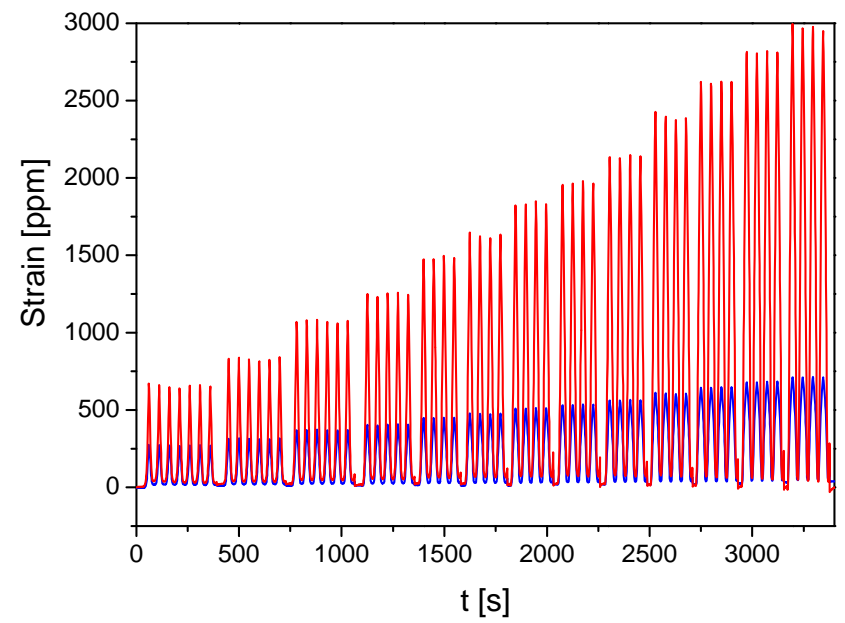

Fig. 12. Longitudinal strain (blue), and amplified strain (red) for a triangular magnetic field of $0.01 \mathrm{~Hz}$ and amplitude from 38.5 to $125 \mathrm{kA} / \mathrm{m}$.

For $125 \mathrm{kA} / \mathrm{m}$ amplitude we measured a strain of 700 ppm for the FBG directly attached to the surface of Terfenol$\mathrm{D}$ rod and 2800 ppm for the FBG connected to the strain amplifier. Fig. 13 shows the strain signal achieved by the strain amplification structure and the strain signal without amplification (directly attached to Terfenol-D) as a response to a triangular magnetic field of $0.01 \mathrm{~Hz}$ and $125 \mathrm{kA} / \mathrm{m}$.

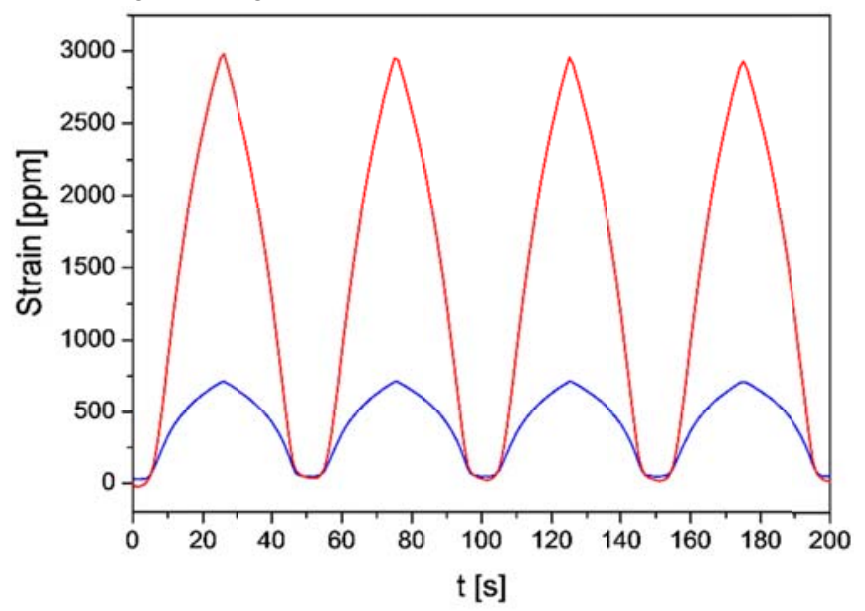

Fig. 13. Longitudinal strain (blue) and amplified strain (red) for a triangular magnetic field of $0.01 \mathrm{~Hz}$ and amplitude of $125 \mathrm{kA} / \mathrm{m}$.

\section{Conclusion}


A magnetic actuator based on Terfenol-D was designed and tested. In order to simultaneously monitor the strain due to magnetization, stress, and temperature, we have implemented a system based on a cylindrical Terfenol-D rod with four FBGs. The magnetostriction was measured in the axial and the transverse direction with two perpendicular FBGs, and the Poisson ratio of the Terfenol-D rod $(v=0,29)$ was calculated on the basis of these measurements. We have used one FBG to measure temperature changes, and another one to measure the thermal expansion of Terfenol-D. The strain due to the Terfenol-D expansion is 2.5 times higher than that of the FBG used for temperature measurement. With this data, the temperature drift of the strain can be compensated. The influence of a compression stress and temperature on the sensitivity of magnetostriction was also investigated. Compression stress increases sensitivity and an increase of temperature reduces the sensitivity. Finally, a strain amplification structure has been designed and fabricated giving opportunity to increase the sensitivity of the strain measurements by a factor of 4 , allowing more accurate control of the magnetostrictive actuator.

\section{References}

[1] Antonio DeSimone and Richard D. James. A theory of magnetostriction oriented towards applications. J. Appl. Phys. 81, (1997) 5706-5708.

[2] Jennera, R.J.E. Smitha, A.J. Wilkinsonb, R.D. Greenough. Actuation and transduction by giant magnetostrictive alloys, A.G., Mechatronics 10 (2000) 457-466.

[3] Debashis Satpathi, Jeffrey A. Moore, Michael G. Ennis. Design of a Terfenol-D Based Fiber-Optic Current Transducer, IEEE SENS. J. , 5, (2005) 1057-1065.

[4] Alcides Oliveira Cremonezi, Elnatan C. Ferreira, Alcino José Biazon Filho, and José A. Siqueira Dias. A Fiber Bragg Grating RMS Current Transducer Based on the Magnetostriction Effect Using a Terfenol-D ToroidalShaped Modulator, IEEE SENS. J., 13, (2013) 683-690.

[5] Verhoeven JD, Gibson ED, McMasters OD, Baker HH. The growth of single crystal terfenoI-D crystals. Metall. Trans. A, 18, (1987) 223-231.

[ 6 ] Grattan KTV, Meggitt BT. Optical fiber sensor technology: advanced applications, Bragg gratings and distributed sensors, Kluwer Academic (2000).

[7] Amat R, García-Miquel H, Barrera D, Kurlyandskaya GV, Sales S (2015), Magneto-optical sensor based on fiber Bragg gratings and a magnetostrictive material. Key Eng. Mat. 644 (2015) 232-235.

[8] Ambrosino C, Campopiano S, Cutolo A, Cusano A. Sensitivity Tuning in Terfenol-D Based Fiber Bragg Grating Magnetic Sensors. IEEE Sensors J., 8, (2008) 1519-1520.
[9] H. Liu, S. Wing Or, H. Yaw Tam. Magnetostrictive composite-fiber Bragg grating (MC-FBG) magnetic field sensor. Sens. Actuators A, 173, (2012) 122-126.

[10] P. Zhang, M. Tang, F. Gao, B. Zhu, S. Fu, J. Ouyang, Z. Zhao, H. Wei, J. Li, P. Ping Shum, and D. Liu. An UltraSensitive Magnetic Field Sensor Based on Extrinsic Fiber-Optic Fabry-Perot Interferometer and Terfenol-D. J. Light. Tech., 33 (2015) 3332-3337.

[11] G. Lanza, G. Bregliob,f, M. Giordanoc,f, A. Gaddid, S. Buontempoe, A. Cusanoa. Effect of the anisotropic magnetostriction on Terfenol-D based fiber Bragg grating magnetic sensors. Sens. Actuators A Phys., 172, (2011) 420-427.

[12] O. Bottauscio, M. Chiampi, A. Lovisolo, P. E. Roccato, and M. Zucca. Dynamic modeling and experimental analysis of Terfenol-D rods for magnetostrictive actuators. J. Appl. Phys. 103, 07F121 (2008).

[13] Hill KO, Meltz G. Fiber Bragg grating technology fundamentals and overview. J. Light. Tech. 15, (1997) 1263-1276. 